\title{
Non-linear Resistivity of a Two-Dimensional Electron Gas in a Magnetic Field
}

\author{
M. G. Vavilov, ${ }^{1}$ I. L. Aleiner, ${ }^{2}$ and L. I. Glazman ${ }^{3}$ \\ ${ }^{1}$ Department of Physics, University of Wisconsin, Madison, WI 53706 \\ 2 Physics Department, Columbia University, New York, NY 10027 \\ ${ }^{3}$ Theoretical Physics Institute, University of Minnesota, Minneapolis, MN 55455
}

(Dated: November 5, 2006)

\begin{abstract}
We develop a theory of nonlinear response to an electric field of a two-dimensional electron gas (2DEG) placed in a classically strong magnetic field. The latter leads to a non-linear current-voltage characteristic at a relatively weak electric field. The origin of the non-linearity is two-fold: the formation of a non-equilibrium electron distribution function, and the geometrical resonance in the inter-Landau-levels transitions rates. We find the dependence of the current-voltage characteristics on the electron relaxation rates in the 2DEG.
\end{abstract}

PACS numbers: 73.40.-c, 73.50.Pz, 73.43.Qt, 73.50.Fq

A magnetic field applied to a two-dimensional electron gas (2DEG) changes the energy spectrum and dynamics of electrons. It leads to a modification of the transport characteristics of the 2DEG even at relatively weak magnetic fields, at which the Landau levels [1] are not resolved yet and the Quantum Hall effect [2] is not developed. The most well-studied modification of that kind is the Shubnikov - de Haas $(\mathrm{SdH})$ oscillations in the resistivity of 2DEG. Its observation, however, is restricted to fairly low temperatures, $T \lesssim \hbar \omega_{\text {c }}$, so that the thermal broadening $T$ of the electron distribution is small compared to the Landau quantization energy $\hbar \omega_{\mathrm{c}}$. In the lowtemperature limit, the strength of the oscillations is controlled by the Dingle factor, $\lambda=\left[-\pi /\left(\omega_{\mathrm{c}} \tau_{0}\right)\right]$; it yields information about the "quantum" lifetime $\tau_{0}$ of the electron [1] due to scattering off disorder.

Recently it was realized [3] that the effect of a magnetic field on the dc non-linear transport, unlike the SdH oscillations of the linear resistivity, is not confined to low temperatures. Oscillations of the differential resistivity with the magnetic field at a finite level of current observed in Ref. [3] persisted to quite high temperatures (about $4 \mathrm{~K}$ ), while the conventional $\mathrm{SdH}$ effect was fully smeared out by temperature. This finding was confirmed in later experiments [4, 5, 6] where the differential resistivity was measured both as a function of the applied magnetic field and transport current. The oscillations of the nonlinear resistance were associated with the geometrical resonance in the electron transitions between the Landau levels [3] that arises from the commensurability of the period in the spatial oscillations of the density of states (DOS) and the diameter $2 R_{\mathrm{c}}$ of an electron cyclotron trajectory. Although this was a plausible explanation of the effect, it remained unclear, why the oscillations are so weakly sensitive to the temperature and which parameters of the $2 \mathrm{DEG}$ control the amplitude of the oscillations.

Another notable effect of magnetic fields on the nonlinear transport in 2DEG was reported in Ref. [5] and deals with the region of relatively small current densities. In that regime, a sharp drop in the differential resistivity was observed. The effect was attributed to the modification of the electron energy distribution caused by the current [7]. Clearly, this modification depends on the energy relaxation rate, and the mech- anisms behind the observations reported in Refs. [3] and [5] seem quite different from each other.

The goal of our work is to show that the two seemingly different phenomena are essentially two manifestations of the electron kinetics described by a standard Boltzmann equation for a weakly disordered 2DEG in the presence of electric and magnetic fields. We demonstrate that the low-current nonlinearity [5] is the consequence of the variation of the occupation factors of electron states: the non-equilibrium population of states renders the transitions normally contributing to the dissipative current ineffective. At high currents, the effect of the electric and magnetic fields on the electron motion becomes important. The oscillations in the $I-V$ characteristic are associated with the geometric resonance in the electron transitions.

We evaluate the dissipative component of the electric current density in a 2 DEG placed in a perpendicular magnetic field $B$ as a function of electric field characterized by the dimensionless parameter $\zeta$,

$$
\zeta=\pi \frac{2 e E R_{\mathrm{c}}}{\hbar \omega_{\mathrm{c}}}, \quad R_{\mathrm{c}}=\frac{v_{\mathrm{F}}}{\omega_{\mathrm{c}}}, \quad \omega_{\mathrm{c}}=\frac{e B}{m_{\mathrm{e}} c},
$$

proportional to the ratio of the work of electric field associated with the displacement of the guiding center of a cyclotron trajectory by $2 R_{\mathrm{c}}$ to the Landau quantization energy $\hbar \omega_{\mathrm{c}}$. The displacement occurs due to the electron scattering off an impurity, and does not exceed $2 R_{\mathrm{c}}$ in a single scattering act. This geometrical constraint leads to the oscillations of the current with $\zeta$; each oscillation corresponds to an increase by $\hbar \omega_{\mathrm{c}}$ of the maximal energy acquired by an electron from the electric field in a single scattering event. The maximal displacement of the guiding center is reached for the scattering angle $\pi$ (backscattering), thus the amplitude of oscillations is proportional to the corresponding scattering rate $1 / \tau(\pi)$.

The "preferred" values of the energy absorbed by an electron from the electric field are multiples of $\hbar \omega_{\mathrm{c}}$ because of the oscillations of the electron DOS associated with the Landau quantization. It is interesting to note however, that even a strong electric field does not result in developing a substantial modulation in the electron energy distribution with the period $\hbar \omega_{\mathrm{c}}$. The reason for that is the dual role electric field plays. On one hand, it promotes the build-up of electron dis- 
tribution at the energies corresponding to the maxima of the DOS. On the other hand, it increases the electron diffusion in energy space, the corresponding coefficient of the spectral diffusion being proportional to the Joule losses. The latter effect wins over the former one, and the electron distribution in energy gets smoother at higher fields. As the result, the oscillatory part of the $I-V$ characteristic reflects the modulation of the electron transition rates with the field, rather the modifications in the electron distribution function; the amplitude of oscillations provides information about the backscattering rate $1 / \tau(\pi)$, hardly accessible in other experiments.

In this work we express the dissipative current in terms of the inelastic relaxation rate $1 / \tau_{\text {in }}$ and harmonics $1 / \tau_{n}$ of the elastic electron scattering rate $1 / \tau(\theta)$ on angle $\theta$ :

$$
\frac{1}{\tau(\theta)}=\sum_{n=-\infty}^{+\infty} \frac{e^{i n \theta}}{\tau_{n}}, \quad \tau_{n}=\tau_{-n}
$$

Typically, the "quantum scattering time" $\tau_{0}$ is short, $\tau_{0} \ll$ $\tau_{\text {in. }}$. However, the transport relaxation time, defined as $1 / \tau_{\text {tr }}=$ $1 / \tau_{0}-1 / \tau_{1}$, may be in an arbitrary relation with $\tau_{\text {in. }}$. We show that the measurements of the dissipative current as a function of $\zeta$ at small $\left(\zeta \lesssim \sqrt{\tau_{0} / \tau_{\text {in }}}\right)$ and large ( $\left.\gtrsim 1\right)$ values of $\zeta$ reveal the rates of inelastic relaxation and of the back-scattering off disorder, respectively.

In the following, we consider the limit of high temperatures $T \gtrsim \hbar \omega_{\mathrm{c}} / 2 \pi^{2}$, when the non-linear resistivity is observed, but the $\mathrm{SdH}$ oscillations are already suppressed [3]. We also limit our analysis to the case of "classically strong" magnetic fields, i.e., we assume that $\omega_{\mathrm{c}} \tau_{0} \lesssim 1$ while $\omega_{\mathrm{c}} \tau_{\mathrm{tr}} \gg 1$. The former condition allows us to keep only the first harmonic in the oscillations of the DOS

$$
v(\varepsilon)=v_{0}\left(1-2 \lambda \cos \frac{2 \pi \varepsilon}{\hbar \omega_{\mathrm{c}}}\right), \quad \lambda=e^{-\pi / \omega_{\mathrm{c}} \tau_{0}} .
$$

The condition $\tau_{\text {tr }} / \tau_{0} \gg 1$ is routinely met in semiconductor heterostructures, and the domain of magnetic fields $1 / \tau_{\text {tr }} \ll$ $\omega_{\mathrm{c}} \ll 1 / \tau_{0}$ is quite wide.

The dissipative part of the electric current

$$
j_{\mathrm{d}}=2 e v_{\mathrm{F}} \int d \varepsilon v(\varepsilon) \int \cos \varphi f(\varepsilon, \varphi) \frac{d \varphi}{2 \pi}
$$

is determined by the stationary electron distribution function $f(\varepsilon, \varphi)$, which is the solution of the following kinetic equation

$$
-\omega_{\mathrm{c}} \partial_{\varphi} f(\varepsilon, \varphi)=\operatorname{St}_{\varphi}\{f(\varepsilon, \varphi)\}+\operatorname{St}_{\mathrm{in}}\{f(\varepsilon, \varphi)\}
$$

Here $\varphi$ is the angle the electron momentum makes with the direction of the electric field. The first term in the right hand side of Eq. (5) is the collision integral for electron scattering off disorder:

$$
\mathrm{St}_{\varphi}\{f\}=\int \frac{v\left(\varepsilon+W_{\varphi \varphi^{\prime}}\right)}{v_{0}} \frac{f\left(\varepsilon+W_{\varphi \varphi^{\prime}}, \varphi^{\prime}\right)-f(\varepsilon, \varphi)}{\tau\left(\varphi-\varphi^{\prime}\right)} \frac{d \varphi^{\prime}}{2 \pi} .
$$

Here $W_{\varphi \varphi^{\prime}}=e E R_{\mathrm{c}}\left[\sin \varphi^{\prime}-\sin \varphi\right]$ is the work of the electric field in the course of the shift $R_{\mathrm{c}} \boldsymbol{z} \times\left[\boldsymbol{n}_{\varphi^{\prime}}-\boldsymbol{n}_{\varphi}\right]$ of the guiding

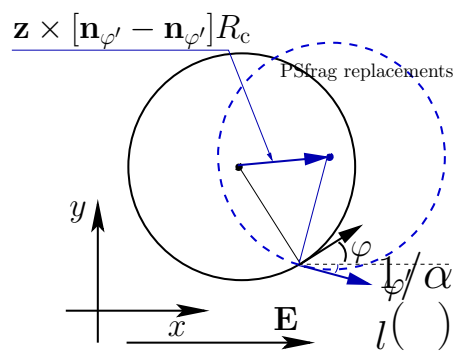

FIG. 1: Electron scattering off impurity changes the momentum direction from $\boldsymbol{n}_{\varphi}$ to $\boldsymbol{n}_{\varphi^{\prime}}$ and the position of the guiding center shifts by $R_{\mathrm{c}} \boldsymbol{z} \times\left[\boldsymbol{n}_{\varphi^{\prime}}-\boldsymbol{n}_{\varphi}\right]$.

center of the cyclotron trajectory, see Fig. 1, unit vector $z$ is perpendicular to the 2DEG plane, and $\boldsymbol{n}_{\varphi}=\{\cos \varphi, \sin \varphi\}$ is directed along the electron momentum. The rate of such scattering events is given by $1 / \tau\left(\varphi^{\prime}-\varphi\right)$ and characterized by its harmonics $1 / \tau_{n}$, see Eq. (2). The imbalance between scattering "in" and "out" terms is determined by the difference of the corresponding distribution functions $f\left(\varepsilon+W_{\varphi \varphi^{\prime}}, \varphi^{\prime}\right)$ and $f(\varepsilon, \varphi)$.

The inelastic relaxation at sufficiently low temperatures is dominated by the electron-electron interaction, represented by the inelastic collision integral

$$
\begin{aligned}
& \operatorname{St}_{\text {in }}\{f(\varepsilon)\}=\int d \varepsilon^{\prime} \int d E M\left(E, \varepsilon, \varepsilon^{\prime}\right) \\
& \times\left[\tilde{f}(\varepsilon) f\left(\varepsilon_{+}\right) \tilde{f}\left(\varepsilon^{\prime}\right) f\left(\varepsilon_{-}^{\prime}\right)-f(\varepsilon) \tilde{f}\left(\varepsilon_{+}\right) f\left(\varepsilon^{\prime}\right) \tilde{f}\left(\varepsilon_{-}^{\prime}\right)\right] .
\end{aligned}
$$

Here $\tilde{f}(\varepsilon) \equiv 1-f(\varepsilon), \varepsilon_{+}=\varepsilon+E, \varepsilon_{-}^{\prime}=\varepsilon^{\prime}-E$ and $M\left(E, \varepsilon, \varepsilon^{\prime}\right)$ describes the dependence of the matrix element of the screened Coulomb interaction on the transferred energy $E$ and the electron energies $\varepsilon$ and $\varepsilon^{\prime}$.

To the first order in $1 / \omega_{\mathrm{c}} \tau_{\text {tr }} \ll 1$ we look for a solution of the kinetic equation Eq. (5) in the form:

$$
\begin{aligned}
& f(\varepsilon, \varphi)=\left[1-\frac{e E \cos \varphi R_{\mathrm{c}}^{2}}{v_{\mathrm{F}} \tau_{\mathrm{tr}}} \partial_{\varepsilon}\right] f_{T}(\varepsilon) \\
& +\lambda\left\{I_{1} \sin \frac{2 \pi \varepsilon}{\hbar \omega_{\mathrm{c}}}+\left[A_{1} \cos \frac{2 \pi \varepsilon}{\hbar \omega_{\mathrm{c}}}+\lambda A_{2}\right] \cos \varphi\right\} \partial_{\varepsilon} f_{T}(\varepsilon) .
\end{aligned}
$$

The first term in Eq. (8) is the result for $\lambda=0$, when the effect of quantization by magnetic field is neglected. The second term in Eq. (8) is proportional to $\lambda$ and oscillates with $\varepsilon / \omega_{\mathrm{c}}$. Here we assume that $T \gg \omega_{\mathrm{c}}$, so that we can separate fast oscillatory dependence on energy of $f(\varepsilon, \varphi)$ with period $\hbar \omega_{c}$ and smooth energy dependence of $f_{T}(\varepsilon)$ on the scale of temperature $T$ of the 2DEG. We assume that $\hbar \omega_{\mathrm{c}} \zeta \ll T$ and that $f_{T}(\varepsilon)$ is close to the Fermi distribution function.

The oscillations amplitude $I_{1}(\varepsilon)$ of isotropic in momentum space component of the distribution function Eq. (8) is determined by

$$
\frac{I_{1}(\varepsilon)}{\tau_{\text {in }}(\varepsilon)} \sin \frac{2 \pi \varepsilon}{\hbar \omega_{\mathrm{c}}}=\left\langle\mathcal{K}_{\varphi \varphi^{\prime}}^{(0)}(\varepsilon) I_{1}+\mathcal{K}_{\varphi \varphi^{\prime}}^{(1)}(\varepsilon)\right\rangle
$$


For the amplitudes $A_{1,2}$ of the anisotropic component in Eq. (8) we have

$$
\begin{aligned}
& \frac{\omega_{\mathrm{c}}}{2} A_{1} \cos \frac{2 \pi \varepsilon}{\hbar \omega_{\mathrm{c}}}=\left\langle\sin \varphi\left[\mathcal{K}_{\varphi \varphi^{\prime}}^{(0)}(\varepsilon) I_{1}+\mathcal{K}_{\varphi \varphi^{\prime}}^{(1)}(\varepsilon)\right]\right\rangle, \\
& \frac{\omega_{\mathrm{c}}}{4} A_{2}=-\left\langle\sin \varphi \cos \frac{2 \pi\left(\varepsilon+W_{\varphi \varphi^{\prime}}\right)}{\hbar \omega_{\mathrm{c}}} \mathcal{K}_{\varphi \varphi^{\prime}}^{(0)}(\varepsilon)\right\rangle I_{1} .
\end{aligned}
$$

Here $\langle\ldots\rangle$ stands for averaging over angle variables $\varphi$ and $\varphi^{\prime}$. The kernels $\mathcal{K}_{\varphi \varphi^{\prime}}^{(0,1)}(\varepsilon)$ are given by

$$
\begin{aligned}
& \mathcal{K}_{\varphi \varphi^{\prime}}^{(0)}(\varepsilon)=\frac{\sin \left[2 \pi\left(\varepsilon+W_{\varphi \varphi^{\prime}}\right) / \hbar \omega_{\mathrm{c}}\right]-\sin \left[2 \pi \varepsilon / \hbar \omega_{\mathrm{c}}\right]}{\tau\left(\varphi-\varphi^{\prime}\right)}, \\
& \mathcal{K}_{\varphi{\varphi^{\prime}}^{\prime}}^{(1)}(\varepsilon)=-2 \cos \frac{2 \pi\left(\varepsilon+W_{\varphi \varphi^{\prime}}\right)}{\hbar \omega_{\mathrm{c}}} \frac{W_{\varphi \varphi^{\prime}}}{\tau\left(\varphi-\varphi^{\prime}\right)} .
\end{aligned}
$$

In the left hand side of Eq. 9a we used the linearized form of the inelastic collision integral Eq. (77). The inelastic relaxation rate $1 / \tau_{\text {in }}(\varepsilon)$ of the oscillating component of the distribution function Eq. (8) was calculated in [7]:

$$
\frac{1}{\tau_{\text {in }}(\varepsilon)}=\frac{\pi^{2} T^{2}+\varepsilon^{2}}{4 \pi E_{\mathrm{F}}} \ln \frac{\kappa v_{\mathrm{F}}}{\max \left\{T, \sqrt{\omega_{\mathrm{c}}^{3} \tau_{\text {tr }}}\right\}},
$$

where $E_{\mathrm{F}}$ is the Fermi energy and $\kappa=4 \pi e^{2} v_{0}$. Solving Eq. (9a) with respect to $I_{1}$, we obtain

$$
I_{1}(\varepsilon)=\frac{-2 e E R_{\mathrm{c}}[d \gamma(\zeta) / d \zeta]}{\tau_{\mathrm{in}}^{-1}(\varepsilon)+\tau_{0}^{-1}-\gamma(\zeta)}, \gamma(\zeta)=\sum_{n} \frac{J_{n}^{2}(\zeta)}{\tau_{n}}
$$

Next we substitute $I_{1}(\varepsilon)$ into Eqs. (9b) and (9c) and find

$$
\begin{aligned}
& A_{1}(\varepsilon)=-\left(2 e E R_{\mathrm{c}} / \omega_{\mathrm{c}} \tau_{\mathrm{tr}}\right)\left[\Gamma_{1}\left(\zeta, \tau_{\mathrm{in}}(\varepsilon)\right)+\Gamma_{2}(\zeta)\right], \\
& A_{2}(\varepsilon)=\left(2 e E R_{\mathrm{c}} / \omega_{\mathrm{c}} \tau_{\mathrm{tr}}\right) \Gamma_{1}\left(\zeta, \tau_{\mathrm{in}}(\varepsilon)\right) .
\end{aligned}
$$

Here we introduced the following notations:

$$
\frac{\Gamma_{1}\left(\zeta, \tau_{\text {in }}\right)}{\tau_{\text {tr }}}=-\frac{[d \gamma(\zeta) / d \zeta]^{2}}{\tau_{\text {in }}^{-1}+\tau_{0}^{-1}-\gamma(\zeta)}, \frac{\Gamma_{2}(\zeta)}{\tau_{\text {tr }}}=-\frac{d^{2} \gamma(\zeta)}{d \zeta^{2}},
$$

and $J_{n}(\zeta)$ are the Bessel functions.

The isotropic in momentum and oscillatory in energy component of the distribution function $f(\varepsilon, \varphi)$ results in the $\Gamma_{1}\left(\zeta, \tau_{\text {in }}\right)$ contribution to the amplitudes $A_{1,2}$ of anisotropic part of $f(\varepsilon, \varphi)$ [7]. The second contribution, containing $\Gamma_{2}(\zeta)$, is coming from the second term in the r.h.s. of Eq. (9b). This contribution was studied in Refs. [8, 9, 10] and originates directly from the effects of electric fields on the collision integral for scattering off disorder.

Substituting the distribution function Eq. (8) with oscillating components Eq. (12) into Eq. (4) and integrating over energy, we obtain the dissipative current:

$$
j_{\mathrm{d}}=\sigma_{\mathrm{D}} E\left[1+2 \lambda^{2} F(\zeta)\right], \quad F(\zeta)=2 \Gamma_{1}\left(\zeta, \tilde{\tau}_{\text {in }}\right)+\Gamma_{2}(\zeta),
$$

where $\sigma_{\mathrm{D}}=e^{2} R_{\mathrm{c}}^{2} \nu_{0} / 2 \tau_{\text {tr }}$ is the Drude conductivity at large Hall angle, $\omega_{\mathrm{c}} \tau_{\mathrm{tr}} \gg 1$. To perform the integration and simplify further analysis we replaced the inelastic relaxation rate $1 / \tau_{\text {in }}(\varepsilon)$ by a parameter $1 / \tilde{\tau}_{\text {in }}$, which can be chosen as $1 / \tilde{\tau}_{\text {in }} \sim 1 / \tau_{\text {in }}(\varepsilon=T)$.

Equation (14) for the dissipative current together with Eqs. (12a) and (13) for functions $\gamma(\zeta), \Gamma_{1}\left(\zeta, \tau_{\text {in }}\right)$ and $\Gamma_{2}(\zeta)$ constitute the central result of the paper. Below we discuss the properties of functions $\Gamma_{1}\left(\zeta, \tilde{\tau}_{\text {in }}\right)$ and $\Gamma_{2}(\zeta)$, which determine the non-linear response of the dissipative current $j_{\mathrm{d}}$, Eq. (14). Then we consider a specific model for $1 / \tau_{n}$ in Eq. (2) and analyze the non-linear behavior of the current within the model.

For weak electric fields, $\zeta \ll 1$, we obtain the following expressions for functions $\Gamma_{1}\left(\zeta, \tilde{\tau}_{\text {in }}\right)$ and $\Gamma_{2}(\zeta)$ expanding the Bessel functions to lowest order in $\zeta^{2}$ :

$$
\begin{aligned}
\Gamma_{1}\left(\zeta, \tilde{\tau}_{\text {in }}\right) & =-\frac{\left(\tilde{\tau}_{\text {in }} / \tau_{\text {tr }}\right) \zeta^{2}}{1+\left(\tilde{\tau}_{\text {in }} / 2 \tau_{\text {tr }}\right) \zeta^{2}}, \\
\Gamma_{2}(\zeta) & =1-\frac{3}{8} \tau_{\text {tr }} \zeta^{2}\left[\frac{3}{\tau_{0}}-\frac{4}{\tau_{1}}+\frac{1}{\tau_{2}}\right] .
\end{aligned}
$$

The $\zeta^{2}$ term in the denominator of Eq. (15a) is legitimate in the limit $\tau_{\text {in }} \gg \tau_{\text {tr }}$, which may take place at sufficiently low electron temperatures. We also note that Eq. (15a) coincides with the result of Ref. [7] in the absence of microwave fields.

In the strong-field limit, $\zeta \gg 1$, we find

$$
\frac{\Gamma_{1}\left(\zeta, \tilde{\tau}_{\text {in }}\right)}{\tau_{\text {tr }}} \propto-\frac{\tau_{0}}{\tau^{2}(\pi)} \frac{\cos ^{2} \zeta}{\zeta^{2}}, \frac{\Gamma_{2}(\zeta)}{\tau_{\text {tr }}} \propto \frac{1}{\tau(\pi)} \frac{\sin 2 \zeta}{\zeta},
$$

where $1 / \tau(\pi)=\sum_{n} e^{i \pi n} / \tau_{n}$ is the back-scattering rate off disorder. The $\Gamma_{2}$-contribution, arising from the effect of electric field on the collision integral, is larger than the $\Gamma_{1}$-contribution, which arises from the stationary out-ofequilibrium component of the distribution function. The latter contribution not only decays faster with the increase of $\zeta$ than the former one, but also contains an additional parameter $\tau_{0} / \tau(\pi)$ which is small for smooth disorder. The amplitude of oscillations of current Eq. (14) decays proportionally to $1 / \zeta$ at $\zeta \gg 1$, but the oscillations in differential conductivity

$$
\sigma=\partial j_{\mathrm{d}} / \partial E \propto \partial\left[\zeta \Gamma_{2}(\zeta)\right] / \partial \zeta \propto \cos 2 \zeta
$$

do not vanish; its maxima and minima are situated at $\zeta=$ $\pi k / 2$ with integer $k$. At smaller values of $\zeta$, term $\Gamma_{1}(\zeta)$ also contributes to $\sigma$ and results in dependence of the oscillations amplitude on $\zeta$ as well as in some shift of maxima and minima from $\zeta=\pi k / 2$.

To discuss the properties of the non-linear current Eq. (14) in a broad range of electric fields, we consider a specific model for the harmonics $1 / \tau_{n}$ of the scattering rate off disordered potential due to charged impurities inside or in the proximity of 2DEG:

$$
\frac{1}{\tau_{n}}=\frac{1}{\tau_{\mathrm{sm}}} \frac{1}{1+\chi n^{2}}+\frac{\delta_{n, 0}}{\tau_{\mathrm{sh}}}, \quad \chi \ll 1
$$

Here $1 / \tau_{\mathrm{sh}}$ is the scattering rate off impurities inside the 2DEG, which produce sharp ( $\delta$-correlated) potential for electrons. Charged impurities in the proximity of $2 \mathrm{DEG}$ produce 


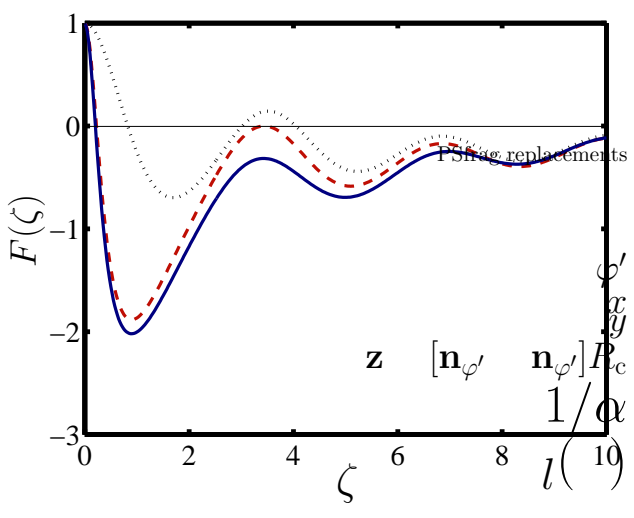

FIG. 2: Function $F(\zeta)$ for disorder described by Eq. 17 with $\tau_{\mathrm{sm}}=\tau_{\mathrm{sh}} / 30$ for several values of $\tilde{\tau}_{\text {in }}$ and $\chi: \tilde{\tau}_{\text {in }}=10 \tau_{\mathrm{sh}}, \chi=0.02$ (solid); $\tilde{\tau}_{\text {in }}=10 \tau_{\text {sh }}, \chi=0.01$ (dashed); $\tilde{\tau}_{\text {in }}=0.5 \tau_{\text {sh }}, \chi=0.01$ (dotted). Note that $F(\zeta)$ has a peak at small $\zeta$ determined by the inelastic relaxation time $\tilde{\tau}_{\text {in }}$ and oscillates at large $\zeta$. At intermediate $\zeta \sim 1$ the positions of maxima and minima of $F(\zeta)$ depend on the relation between various parameters of the model.

a smooth potential resulting in electron scattering on small angle $\theta \sim \sqrt{\chi} \ll 1$, where $\chi$ can be estimated as $\chi \sim\left(\lambda_{\mathrm{F}} / \xi\right)^{2}$, with $\lambda_{\mathrm{F}}$ and $\xi$ being the Fermi wavelength and the correlation length of the disorder potential, respectively. The two restrictions for validity of Eq. (14) on the strength of magnetic field $\left(\omega_{\mathrm{c}} \tau_{\text {tr }} \gg 1\right.$ and $\left.\omega_{\mathrm{c}} \tau_{0} \lesssim 1\right)$ can be satisfied simultaneously for $\chi \ll 1$.

For the disorder characterized by harmonics of the scattering rate Eq. (17), we obtain $\gamma(\zeta)=J_{0}^{2}(\zeta) / \tau_{\mathrm{sh}}+$ $1 /\left(\tau_{\mathrm{sm}} \sqrt{1+\chi \zeta^{2}}\right)$. Here we omitted the term, which arises from the back-scattering off smooth disorder and yields exponentially small $(\exp (-\pi / \sqrt{\chi})$ ) contribution to $1 / \tau(\pi)$, cf. Eq. (16). Substituting this expression for $\gamma(\zeta)$ in Eq. (13), we can evaluate the current Eq. (14) at arbitrary $\zeta$. At $\zeta \ll 1 / \sqrt{\chi}$ we have

$$
\frac{F(\zeta)}{\tau_{\mathrm{tr}}}=\frac{1}{\tilde{\tau}_{\mathrm{sm}}}-\frac{2\left[2 J_{0}(\zeta) J_{1}(\zeta) / \tau_{\mathrm{sh}}+\zeta / \tilde{\tau}_{\mathrm{sm}}\right]^{2}}{\tilde{\tau}_{\mathrm{in}}^{-1}+\tau_{\mathrm{sh}}^{-1}\left[1-J_{0}^{2}(\zeta)\right]+\tilde{\tau}_{\mathrm{sm}}^{-1} \zeta^{2} / 2}-\frac{\left[J_{0}^{2}(\zeta)\right]^{\prime \prime}}{\tau_{\mathrm{sh}}},
$$

where $1 / \tilde{\tau}_{\mathrm{sm}}=\chi / \tau_{\mathrm{sm}}$ is the smooth disorder contribution to the transport scattering rate. Equation (18) covers both the regime of relatively weak fields, where inelastic scattering is important, and the regime of strong fields, exhibiting prominent oscillations. Function $F(\zeta)$ has a sharp feature at $\zeta \sim \sqrt{\tau_{\mathrm{sh}} / \tau_{\mathrm{in}}}$. At these fields, the spectral diffusion of electrons caused by electric field becomes comparable with the inelastic relaxation. At stronger fields, $\zeta \gtrsim \sqrt{\tilde{\tau}_{\mathrm{sm}} / \tau_{\mathrm{in}}}$, the two kinds of disorder, smooth and "sharp", yield two separate contributions to $F(\zeta)$,

$$
\frac{F(\zeta)}{\tau_{\mathrm{tr}}}=\frac{1-3 \chi \zeta^{2}-2\left(1+\sqrt{1+\chi \zeta^{2}}\right)}{\tilde{\tau}_{\mathrm{sm}}\left(1+\chi \zeta^{2}\right)^{5 / 2}}-\frac{\left[J_{0}^{2}(\zeta)\right]^{\prime \prime}}{\tau_{\mathrm{sh}}} .
$$

Here only the sharp component of disorder contributes to the oscillatory behavior of $F(\zeta)$.

We calculated the dissipative component of electric current in response to the applied dc electric field of arbitrary strength within self-consistent Born approximation [1]. We show that the non-linear component of the current consists of two contributions. One contribution arises due to the formation of the out-of-equilibrium component of the distribution function, oscillating as a function of energy. The second contribution is the result of modification by electric field of electron scattering amplitudes off the disorder potential. We showed that the former contribution is important at relatively weak fields, while the latter one dominates in the high-field domain. There, the non-linear contribution to the current oscillates as a function of the applied electric field. The amplitude of oscillations of the differential conductivity does not decrease with the increase of electric field (and at fixed magnetic field). It may be necessary to take into account the effect of heating on the quantum scattering time and thus on the Dingle factor $\lambda$ in order to explain the suppression of oscillations observed in [6].

Finally, we considered the limit $\omega_{\mathrm{c}} \tau_{0} \lesssim 1$, and therefore assumed that the oscillations in the DOS are described by one harmonic with period in energy $\hbar \omega_{\mathrm{c}}$, Eq. (3). In stronger fields, the DOS remains periodic in energy with the same period $\hbar \omega_{c}$, but contains higher harmonics [1, 8]. These higher harmonics in the DOS result in a more complicated form of the oscillatory part of the non-linear resistivity.

The authors are thankful to S. Vitkalov and M. Zudov for discussions of experiments. Part of this work was performed during the visit to the Aspen Center for Physics. The work was supported by the W. M. Keck Foundation and NSF grants DMR 02-37296, DMR 04-39026 and DMR-0408638.

[1] T. Ando, A. Fowler, and F. Stern, Rev. Mod. Phys. 54, 437 (1982).

[2] R. E. Prange and S. M. Girvin, The Quantum Hall Effect, 2nd ed. (Springer, ADDRESS, 1990).

[3] C. L. Yang, J. Zhang, R. R. Du, J. A. Simmons and J. L. Reno, Phys. Rev. Lett. 89, 076801 (2002).

[4] A. A. Bykov, J. Zhang, Sergey Vitkalov, A. K. Kalagin and A. K. Bakarov, Phys. Rev. B 72, 245307 (2005).

[5] J. Zhang, S. Vitkalov, A. A. Bykov, A. K. Kalagin and A. K. Bakarov, cond-mat/0607741.

[6] W. Zhang, H. -S. Chiang, M. A. Zudov, L. N. Pfeiffer and K. W. West, cond-mat/0608727.

[7] I. A. Dmitriev, M. G. Vavilov, I. L. Aleiner, A. D. Mirlin and D. G. Polyakov, Phys. Rev. B 71, 115316 (2005).

[8] M. G. Vavilov and I. L. Aleiner, Phys. Rev. B 69, 035303 (2004).

[9] A. C. Durst, S. Sachdev, N. Read, and S. M. Girvin, Phys. Rev. Lett. 91, 086803 (2003).

[10] V. I. Ryzhii, R. A. Suris, and B. Shchamkhalova, Sov. Phys. Semicond. 20, 1299 (1986). 